\title{
Surgical Site Infection: The Clinical and Economic Impact
}

\author{
Megan C. Turner, MD ${ }^{1}$ John Migaly, MD FACS, FASCRS² \\ ${ }^{1}$ Department of Surgery, Duke University Medical Center, Durham, \\ North Carolina \\ 2 Division of Advanced $\mathrm{GI}$ and Oncologic Surgery, Duke University \\ Medical Center, Durham, North Carolina \\ Address for correspondence Megan C. Turner, MD, Department of \\ Surgery, Duke University Medical Center, 7674 HAFS Building, DN, \\ Erwin Road, Durham, NC 27710 \\ (e-mail: Megan.Turner@duke.edu).
}

Clin Colon Rectal Surg 2019;32:157-165.

\begin{abstract}
Keywords

- surgical site infection

- economic impact of SSI

- surgical site infection prevention bundles

- cost

- complications

Surgical site infection ( $\mathrm{SSI}$ ) following colorectal surgery is associated with worse postoperative outcomes, longer length of stay, and higher rates of readmission. SSI rates have been established as a surrogate metric for the overall quality of surgical care and are intricately tied to financial incentives and the public reputation of an institution. While risk factors and prevention mechanisms for SSI are well established, the rates of SSI remain high. This article discusses the clinical and economic impact of SSI and strategies for mitigating the risk of SSI through bundled prevention practices.
\end{abstract}

Modern germ theory rejects the historical surgeon's reliance on "laudable pus" as an indication of healing from surgical injury. The prevention of surgical site infection (SSI) is increasing in clinical and economic relevance. Mechanisms to predict and prevent SSI pervade the literature, yet the economic impact of SSI and implementation of prevention programs have been incompletely described. Colorectal surgeons are leading the movement to improve rates of SSI and their work is of interest to other surgeons, patients, hospitals, and payer groups. This article will discuss current evidence and practice surrounding clinical implications of superficial SSI, the economic impact of these infections, and strategies for mitigating this complication through effective implementation of standardized care bundles.

SSI has always been of interest to surgeons and patients, and the development of quality improvement metrics and reporting has allowed scientific investigation to occur at a national scale. Risk factors associated with SSI include patient comorbidities, intraoperative factors, and postoperative wound management. Poor outcomes associated with SSI include increased morbidity, length of hospital stay, readmission, and mortality. Patients undergoing colorectal surgery are disproportionately affected by $\mathrm{SSI}^{1}$ with rates of up to $25 \%$, and up to $55 \%$ of these infections have been deemed preventable. ${ }^{2,3}$ The importance of prevention of SSI is underscored with the incorporation into national guidelines and financial penalties.

In addition to the association with poor clinical outcomes, high SSI rates are associated with negative economic consequences. ${ }^{3}$ A longer hospital stay represents a direct cost to the hospital system and the payer, but also has patient and societal economic implications from cost of supplies and nursing care, to extended loss of work/productivity. In light of this, financial incentives and penalties are associated with SSI outlier status through Centers for Medicare and Medicaid Services (CMS).

Bundled SSI prevention practices are well characterized in the literature, ${ }^{4-6}$ with the aggregate effect of the bundle exceeding the isolated impact of each component. While conceptually well accepted among colorectal surgeons, cost, variability in bundle components, and barriers to implementation have stunted widespread adoption. Despite current bundles being developed and incorporated in academic institutional silos, increasing data regarding efficacy and cost-effectiveness will likely promote uptake within all hospital types.
Issue Theme Complications and Dilemmas in Colorectal Surgery; Guest Editor: Skandan Shanmugan, MD
Copyright $\odot 2019$ by Thieme Medical Publishers, Inc., 333 Seventh Avenue, New York, NY 10001, USA. Tel: +1(212) 584-4662.
DOI https://doi.org/ 10.1055/s-0038-1677002. ISSN 1531-0043. 


\section{Clinical}

Development of quality initiatives has garnered national support ${ }^{7}$ following the 1999 publication To Err is Human: Building a Safer Health System ${ }^{8}$ and increasing public attention toward preventable harm in the hospital setting. SSI has become the surrogate for surgical quality at large leading to programs for institutional monitoring and benchmarking SSI rates. As early as the 1970s, the Center for Disease Control and Prevention began the National Nosocomial Infections Surveillance (NNIS) program which allowed for voluntary and confidential reporting of hospital-acquired infections (HAIs), and allowed for interhospital comparison. ${ }^{9}$ In the 1980s, the Veterans Affairs (VA) Hospitals was scrutinized for its surgical quality compared with the private sector. Given that quality outcomes for comparison were not widely available, projects to fill this process gap were promoted. As a result of the VA studies, the American College of Surgeons (ACS) National Surgical Quality Improvement Project (NSQIP) was developed in 1994 to provide data tracking and analytics for postoperative complications. The program was expanded to the private sector in 1999. In 2005, NNIS was merged with other smaller programs into the National Healthcare Safety Network (NHSN) with the intention of increasing ease and accuracy of reporting, with the intention of using surveillance and benchmarks as a mechanism for quality improvement. ${ }^{10}$ In contrast to NHSN, the NSQIP generates data from patient charts, inclusive of 30-day inpatient and outpatient outcomes, and is risk adjusted for comparisons. ${ }^{11}$ While these programs use different methods of data acquisition, ${ }^{12,13}$ monitoring within an institution can occur over time and comparisons can be made between institutions. The programs allow for comparison of the success of quality improvement programs both within institutions overtime and nationally.

Early research on the negative impact of SSI on postoperative recovery and the development of programs to follow rates of SSI stimulated the 2002 Surgical Care Improvement Project measures to be developed and implemented. ${ }^{14,15}$ These measures led to the Joint Commission best practice guidelines, which have most recently been updated in 2014. ${ }^{16}$ These guidelines are presented in the publication A Compendium of Strategies to Prevent Healthcare Associated Infections in Acute Care Hospitals and include parenteral preoperative antibiotics 1 hour prior to incision, antibiotic coverage consistent with published guidelines, antibiotics discontinued within 24 hours of surgery, normothermia, standardization of skin preparation, and patient and family education. ${ }^{16}$ The Joint Commission Center for Transforming Health Care reported a 32\% reduction in SSI, and a savings of $\$ 3.7$ million following implementation of their guidelines emphasizing the benefit of attention to prevention of SSI. ${ }^{17,18}$

Concerns regarding the validity of each program for monitoring SSI have been outlined. Specific concerns regarding NHSN and Agency for Healthcare Research and Quality (AHRQ) measures include the use of unadjusted rates and exclusive inclusion of inpatient infections. These concerns are relevant in that complex subspecialty practices may have higher rates of SSI than general elective community practices, ${ }^{19}$ and that the inpatient assessment dramatically underrepresents SSI, up to half of which are diagnosed in the outpatient setting. ${ }^{19,20}$ Despite 30-day inpatient and outpatient follow-up, NSQIP captures only a sample of cases, and these are exclusively from participating institutions. Additionally, the caliber of documentation must be monitored closely, as Speicher et al note in their discussion of SSI following hepatopancreaticobiliary (HPB) surgery "seemingly modest changes in documentation can lead to substantial changes in perceived outcomes and performance" for SSI. ${ }^{21}$ Attempts to adjust for complexity of case and patient populations predisposed to SSI have led to rigorous research of SSI predictors and their associated outcomes.

Predictors of SSI following colorectal surgery have been established and validated. Preliminarily, the NNIS SSI risk index of the late 20th century created an operation-specific score utilizing American Society of Anesthesiologists class, wound classification, and operative duration. ${ }^{19}$ Subsequently, through retrospective large database analysis and prospective studies, more granular measures of SSI risk have been developed. ACS NSQIP developed a risk calculator to assess a patient's risk for postoperative complications in the clinic setting. Included variables are demographics, comorbidities, and procedure variables. ${ }^{11,22}$ Key unmodifiable predictors of increased risk include contaminated and dirty wound classification, multiple comorbidities, ${ }^{1}$ and obesity. ${ }^{1,20,23}$ Potentially modifiable factors are targeted in guidelines ${ }^{24}$ and bundled prevention practices and include bowel preparation with antibiotics, ${ }^{25}$ appropriate timing and coverage of parenteral antibiotics, ${ }^{14,26,27}$ use of chlorhexidine skin preparation, glucose control, ${ }^{28}$ intraoperative normothermia, ${ }^{29,30}$ appropriate oxygenation, ${ }^{30-32}$ and open compared with laparoscopic surgery. ${ }^{20}$ Substantial evidence exists for mechanical bowel preparation with enteral antibiotics to minimize the risk of SSI for patients undergoing colorectal surgery. ${ }^{25}$ Infusion of broad-spectrum antibiotics such as ciprofloxacin with metronidazole, or ertapenem within an hour of incision, ${ }^{23,33}$ with appropriate weight base dosing, and time-appropriate re-dosing is imperative to minimize risk of SSI. ${ }^{14,26,27,33}$ Standardized chlorhexidine skin preparation has been shown to decrease SSI rates, and is included in many prevention bundles. Glycemic control in the intraoperative and postoperative period has been incorporated into cardiac surgery guidelines, and is increasingly being recognized in colorectal surgery as a key factor in minimizing SSI risk. ${ }^{28}$ Normothermia and prevention of hypothermia with forced warm-air heating blankets are associated with decreased SSI risk. ${ }^{29,30}$ Minimally invasive techniques provide a $50 \%$ reduction in postoperative SSI compared with open surgery, and are becoming the standard of care in colorectal surgery. While these components have been implemented with variable uptake, bundled SSI prevention practices are being shown to be of greater efficacy than the isolated components. This is described in detail later.

Concurrent with studies to identify predictors, and therefore modify and prevent SSI in colorectal surgical patients, 


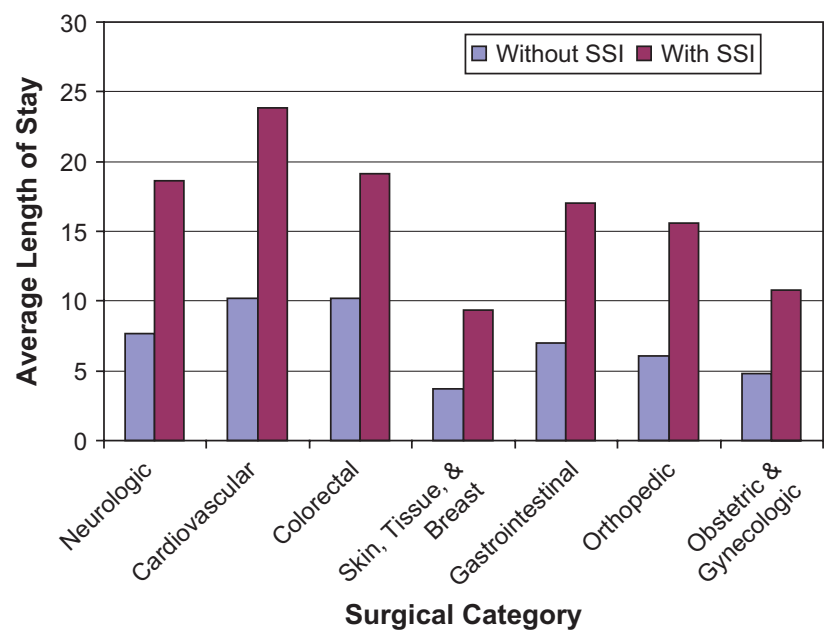

Fig. 1 Impact of surgical site infection (SSI) on length of stay, showing increased length of stay in patients with SSI (de Lissovoy et al in 2009).

additional studies have established the impact of SSI on clinical outcomes. While clear associations between SSI and adverse outcomes are present, quantifying the impact on additional outcomes is relevant to prognosticating how patients will do in the postoperative period. Length of stay and features of readmission were important in establishing the cost of SSI, therefore allowing analytics regarding costeffectiveness of different prevention programs. Adult patients diagnosed with SSI during their inpatient hospitalization have a longer length of stay compared with unaffected counterparts (9.5 vs. 8.1 days $^{20}$ ) ( - Fig. 1 ). This has been demonstrated in pediatric populations as well, with an increase of 10 hospital days and an increase of $\$ 27,000$ in costs. ${ }^{34}$ However, nearly $50 \%$ of SSIs are diagnosed in the outpatient setting. This phenomenon leads to variable rates of reported SSI based on the clinical data extraction technique: AHRQ versus NSQIP versus internal institutional monitoring programs as described earlier. Additionally, outpatient SSI diagnoses are associated with readmissions at a higher frequency than unaffected counterparts ( $27.8 \mathrm{vs.}$ $\left.6.8 \%{ }^{20}\right), 35$ and these readmissions are on average 2 days longer in duration than readmissions for other causes. ${ }^{20}$

Preventative measures incorporated at the institutional and national level include appropriate choice and timing of prophylactic antibiotics, perioperative hair clipping, glucose control, and normothermia. ${ }^{2,15,24,36,37}$ Despite the development of standardized quality measures, including best practice guidelines from the Center for Disease Control and Prevention $^{38}$ and the World Health Organization, ${ }^{39}$ adherence to individual guidelines alone has not reduced the patient harm caused by SSI to an acceptable level. ${ }^{15,24,37}$ In light of this, alternate methodologies to improve SSI rates have been implemented. ${ }^{24}$ Enhanced recovery pathways and bundled SSI reduction protocols have become common in colorectal surgery groups in an attempt to improve postoperative outcomes. ${ }^{40-43}$ During this time, minimally invasive surgical technique has demonstrated substantial improvements in SSI rates compared with open procedures with reductions as high as $60 \%$ with use of minimally invasive surgery compared with open surgical techniques. ${ }^{44,45}$ Bundle protocols are proposed as a cost-effective mechanism for further reduction in SSI when implemented in aggregate. ${ }^{40,46,47}$

\section{Economic}

The true cost of SSI is difficult to quantify given variations in methodology utilizing inconsistent intrinsic and extrinsic costs. ${ }^{48,49}$ National estimates reach more than $\$ 3$ billion dollars annually. ${ }^{50,51}$ Intrinsic costs of SSI to patient care are in the management of the infection: additional operations, procedures, nursing, wound management personnel time, infectious disease interventions, loss of time from work, and costs associated with home health. ${ }^{20,52,53}$ Extrinsic costs are substantial, including time away from work, functional decline, litigation, the reputation of the medical center, and the impact of variable reimbursement and penalties based on performance. ${ }^{52}$ Additional costs accrued by patients with SSI have been reported in a wide range from $\$ 1,400$ to $\$ 40,500^{50}$ with superficial SSI accruing less cost than deep space infections. In 2013, Zimlichman et al estimated the additional cost of SSI to be $\$ 20,785$ (95\% confidence interval: $\$ 18,902-\$ 22,667)$ per occurrence. ${ }^{51}$ This is comparable to the estimates by de Lissovoy et al (2009), $\$ 20,842$ ( - Fig. 2), ${ }^{1}$ and Wick et al (2011), $\$ 14,608 .{ }^{20}$ Patients accept variable out-of-pocket costs, but estimates around $\$ 6,200$ have been proposed for the cost of home wound management of SSI ${ }^{19}$ which further emphasizes the need for effective prevention programs. Despite heterogeneity in methodology to determine costs, programs to optimize postoperative care such as enhanced recovery pathways and SSI bundles have been evidence to support costeffectiveness. ${ }^{40,54}$

Quality-based reimbursements and penalties are associated with rates of SSI, specifically those deemed to be HAIs

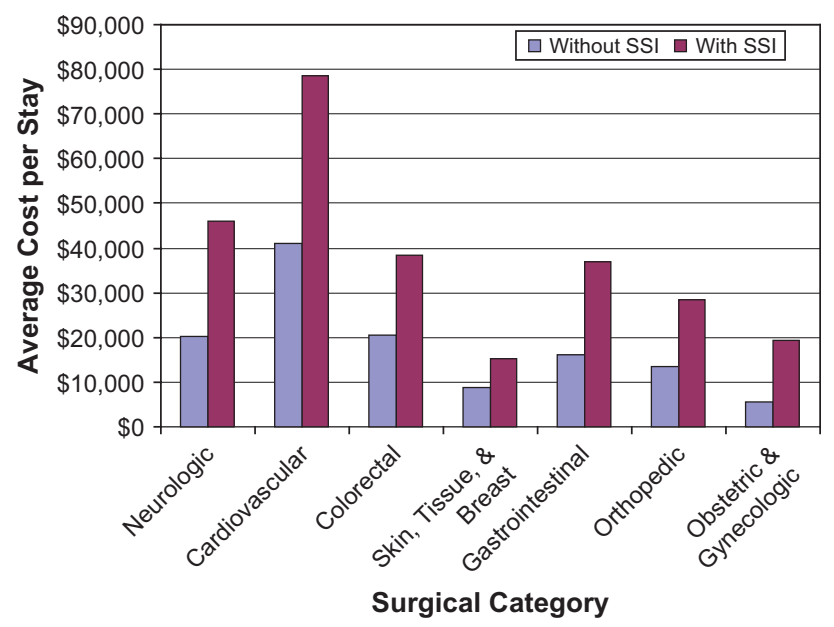

Fig. 2 Impact of surgical site infection (SSI) on cost, showing increased cost for patients experiencing SSI across specialties (de Lissovoy et al in 2009). 


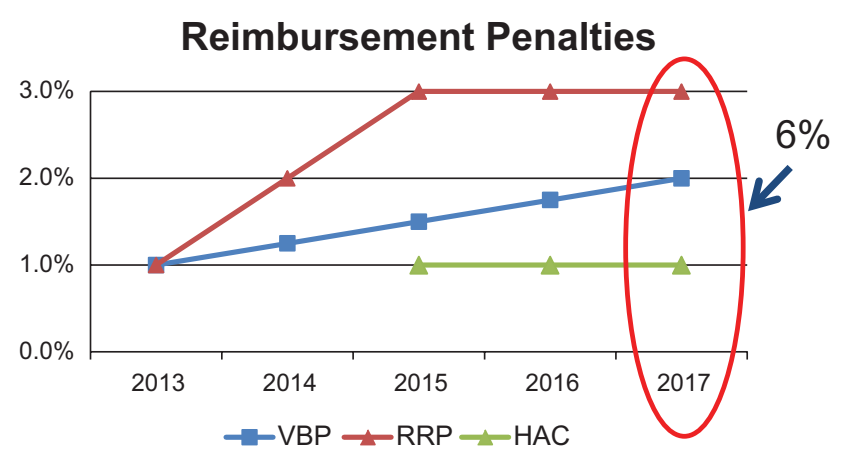

Fig. 3 Reimbursement penalties from Center Medicare/Medicaid Services. HAC, hospital-acquired condition; RRP, readmission reduction program; VBP: value-based payment.

by the CMS. ${ }^{55}$ CMS assigns financial penalties of up to $6 \%$ of reimbursement for institutions not meeting the compliance metrics outlined in best practice guidelines ( $\mathbf{F i g . ~ 3 ) . 5 6 , 5 7}$ Additionally, CMS has reduced reimbursements to institutions that fall below $25 \%$ in their quality measure score, calculated using rates of central line-associated bloodstream infection, catheter-associated urinary tract infection, SSI (colon surgery and abdominal hysterectomy), methicillinresistant Staphylococcus aureus bacteremia, and Clostridium difficile infection. ${ }^{57}$ Prior CMS reimbursement penalties have been effective in reducing poor outcomes in hospitalized patients. HAIs were reduced by 10\% following changes to reimbursement. ${ }^{58}$ However, other hospital-acquired conditions such as pressure ulcers and falls did not have associated decreases following reimbursement changes. ${ }^{58}$ While enthusiasm for medical cost containment drives the development of policies such as those outlined earlier, prior analysis of CMS reimbursement withholdings in the settings of complications has shown only modest national cost savings. ${ }^{58,59}$ Alternate strategies to pay-for-performance such as pay-forparticipation may ultimately lead to novel improvements in surgical quality. ${ }^{60,61}$ However, the evidence toward improved quality in conjunction with modest financial improvements will likely garner enough support to maintain the pay-for-performance guidelines.

\section{Prevention Bundles}

Robust literature exists to support bundled SSI prevention practices as a mechanism to improve SSI rates. While bundled practices are becoming conceptually accepted among colorectal surgeons, substantial perceived barriers to implementation remain. ${ }^{62}$ Previous studies stress the formation of multidisciplinary teams as a key component in establishing process improvement. ${ }^{24,63,64}$ Additionally, iterative changes performed in the multidisciplinary setting have improved guideline adherence, decreased the SSI rate, and decreased emergence of resistant organisms. ${ }^{24,64}$

In 2007, Hedrick et al described success in the implementation of a multifactorial colorectal wound management protocol at a single institution. ${ }^{5}$ In their study, a $39 \%$ reduc- tion in SSI followed implementation of their bundle, which included features of appropriate antibiotic administration and timely discontinuation, normothermia, and perioperative glucose management. Improvements in compliance in three of the components were observed. Additionally, the success observed in colorectal SSI bundle implementation also led to improvements of SSI rates in other intra-abdominal operative cohorts at their institution.

Following early successes, Bull et al described similar bundle implementation at a single institution in Australia. Their protocol included implementing protocols for intraoperative normothermia, fraction of inspired oxygen delivery goals intraoperatively, systolic blood pressure goals, glycemic control, and appropriate antibiotic prophylaxis (-Fig. 4). ${ }^{4}$ Overall, they saw low compliance with each specific measure; however, they saw a measurable improvement in the SSI rate. Their institution identified barriers to the implementation of staff education and turnover, and resource utilization which were partially overcome by the engagement of a project champion who, over time, improved the institutional adherence to the protocol.

In 2014, Keenan et al described a large single institution SSI prevention bundle implementation project with preoperative, intraoperative, and postoperative protocol described later (-Fig. 5). ${ }^{6}$ Their study demonstrated marked improvements on univariate analysis in superficial wound infection ( 24.9 vs. $5.6 \%, p<0.001)$, sepsis $(10.4$ vs. $2.3 \%$, $p<0.001$ ), and length of stay ( 6 vs. 5 days, $p<0.001$ ). Additionally, they established the $35 \%$ higher variable direct costs associated with SSI occurrence. Their SSI prevention bundle components preoperatively included a chlorhexidine shower, a mechanical bowel preparation with oral antibiotics, ertapenem as the parenteral antibiotics of choice 1 hour prior to incision, and field preparation with chlorhexidine alcohol. Intraoperatively, it featured the use of a fascial wound protector, a gown and glove change prior to fascial closure, a dedicated wound closure tray, and limited operating room traffic to key personnel. As in prior protocols, intraoperative and postoperative time periods, euglycemia, and normothermia were maintained. Postoperatively, the key features of the bundle include removal of the sterile dressing within 48 hours and daily washing of the incision with chlorhexidine. Across all phases of care, education regarding SSI prevention was reinforced to patients, families, and care providers.

Most recently, in 2017, Vu et al presented their encouraging regional data from the Michigan Collaborative. They demonstrated multi-institutional improvements in postoperative outcomes through the increased uptake of three features of their recommended SSI prevention bundle. ${ }^{65}$ The Michigan Surgical Quality Collaborative provided funding to participating institutions to implement their bundle in an autonomous fashion with focus on preoperative parenteral antibiotics, mechanical bowel preparation with enteral antibiotics, and normoglycemia from 2012 to 2016. Over the study period, they increased adherence to the components of the bundle, and decreased their SSI rate from 6.7 to $3.9 \%$ $(p<0.05)$ ( - Figs. 6 and 7$).{ }^{65}$ Their experience provides 
Bundle of care for patients undergoing colorectal surgery

\begin{tabular}{|c|c|}
\hline Bundle component & Comments \\
\hline $\begin{array}{l}\text { Temperature maintained } \\
\geq 36{ }^{\circ} \mathrm{C} \text { peri-operatively } \\
\text { and for } 1 \mathrm{~h} \text { postoperatively }\end{array}$ & $\begin{array}{l}\text { Recommendations included documentation } \\
\text { of temperature, use of warmed blankets } \\
\text { pre- and postoperatively, use of Bair Huggers } \\
\text { and warmed fluids intra-operatively }\end{array}$ \\
\hline $\begin{array}{l}\text { Fraction of inspired oxygen } \\
\text { delivered maintained } \\
\geq 0.8 \text { intra-operatively; } \\
\text { adequate oxygenation } \\
\text { for } 4 \text { h postoperatively }\end{array}$ & $\begin{array}{l}\text { Adequate postoperative oxygenation was } \\
\text { defined initially as administration of at least } \\
6 \mathrm{~L} \text { oxygen/min and regular monitoring of } \\
\text { oxygen saturation. Later in the project, this } \\
\text { was changed to use of a high-flow } \\
\text { non-rebreathing mask for } 4 \mathrm{~h} \text { postoperatively }\end{array}$ \\
\hline \multicolumn{2}{|l|}{$\begin{array}{l}\text { Systolic blood pressure } \\
\text { maintained } \geq 90 \mathrm{mmHg} \\
\text { intra- and postoperatively }\end{array}$} \\
\hline $\begin{array}{l}\text { Blood sugar level maintained } \\
\leq 10 \text { mmol pre- and } \\
\text { intra-operatively }\end{array}$ & $\begin{array}{l}\text { Documentation of pre- and intra-operative } \\
\text { blood sugar level was requested from } \\
\text { February } 2009\end{array}$ \\
\hline $\begin{array}{l}\text { Appropriate antibiotic } \\
\text { prophylaxis given }\end{array}$ & $\begin{array}{l}\text { Appropriate choice, timing and second } \\
\text { dose for prolonged procedures }(>3 \mathrm{~h})\end{array}$ \\
\hline
\end{tabular}

Fig. 4 Surgical site infection prevention bundle described by Bull et al (2011).

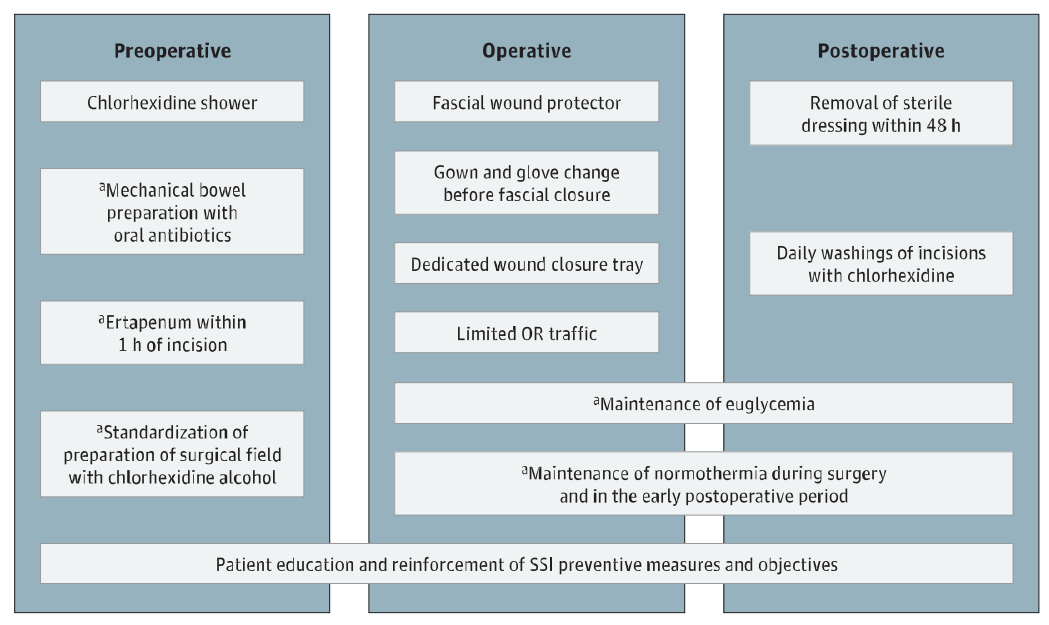

Fig. 5 Surgical site infection prevention bundle described by Keenan et al (2014).

evidence for success of regional implementation with significant improvement in SSI, where prior studies were intuition specific.

A single institution utilized their 30-day NSQIP data to compare SSI in colorectal surgical patients, following implementation of an enhanced recovery pathway and a SSI prevention bundle. ${ }^{40}$ The authors found a reduction in infection rates and a reduction in overall cost for colorectal surgical admissions. Following implementation of an enhanced recovery pathway, they saw a reduction in length of stay, which frequently is used as a cost surrogate. The implementation of the SSI prevention bundle decreased rates of superficial site infection ( 16.1 vs. $6.3 \%, p<0.01$ ) and sepsis $(11.2$ vs. $1.8 \%, p<0.01)$. While some overhead costs exist in the implementation of such programs, overall cost for admissions can be reduced (\$31,926 in 2008 vs. $\$ 22,044$ in $2013, p<0.01) .^{40}$ This study expands on the previous efficacy study of bundled SSI prevention and enhanced recovery pathways. ${ }^{5,6,66}$ Their experience demonstrates feasible, cost-effective improvements in SSI rates with successful implementation of comprehensive perioperative pathways (-Fig. 8). 


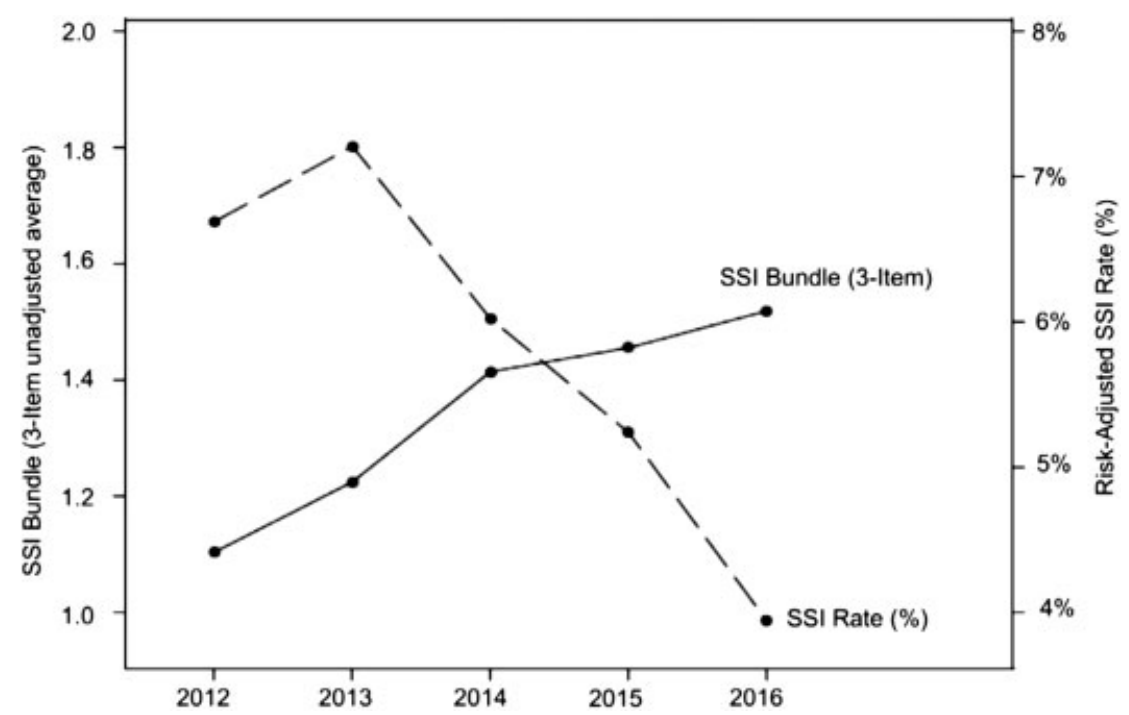

Fig. 6 Impact surgical site infection prevention bundle by Michigan Collaborative described by Vu et al (2017).

\section{Conclusion}

SSI remains a critical metric for quality of colorectal surgical care. SSI rates are inextricably related to patient and institution, clinical and financial outcomes, and prevention is of paramount importance. Refining clinical predictors associated with SSI will allow for appropriate counseling of patients and improved risk adjustment in reporting SSI rates. Granular cost data are important in developing national and institutional budgets and appropriate reimbursements and penalties. Bundled SSI prevention practices in aggregate are promising in reducing SSI rates to below what can be achieved through isolated guidelines alone, and while individual institutions have shown promise in both clinical and financial outcomes, widespread implementation remains elusive. Further research in implementation sciences and the development of policy to maximize prevention strategies will be required to best serve the patients of the future.

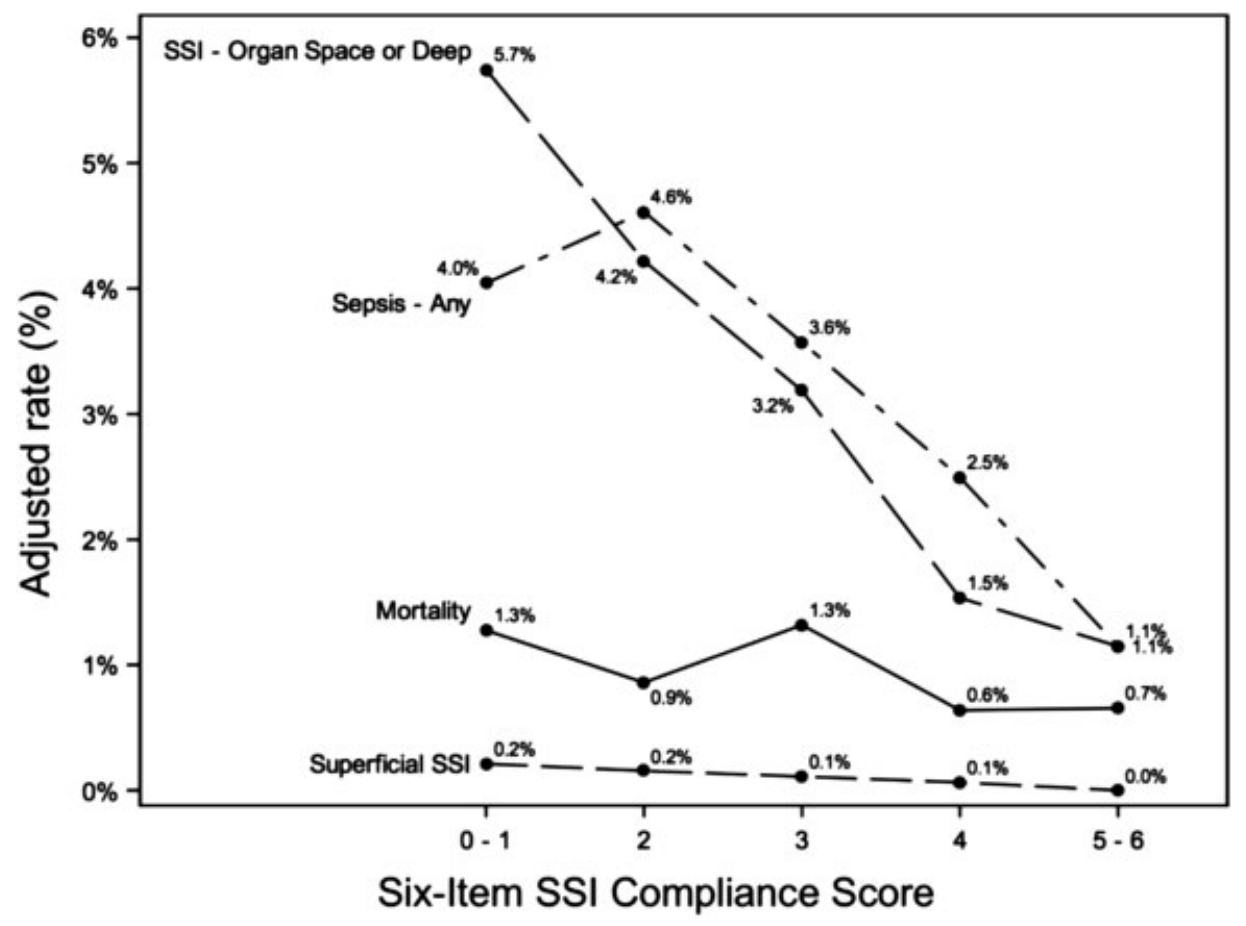

Fig. 7 Postoperative complication rates and surgical site infection rates by surgical site infection (SSI) prevention bundle compliance score (Vu et al, 2017). 


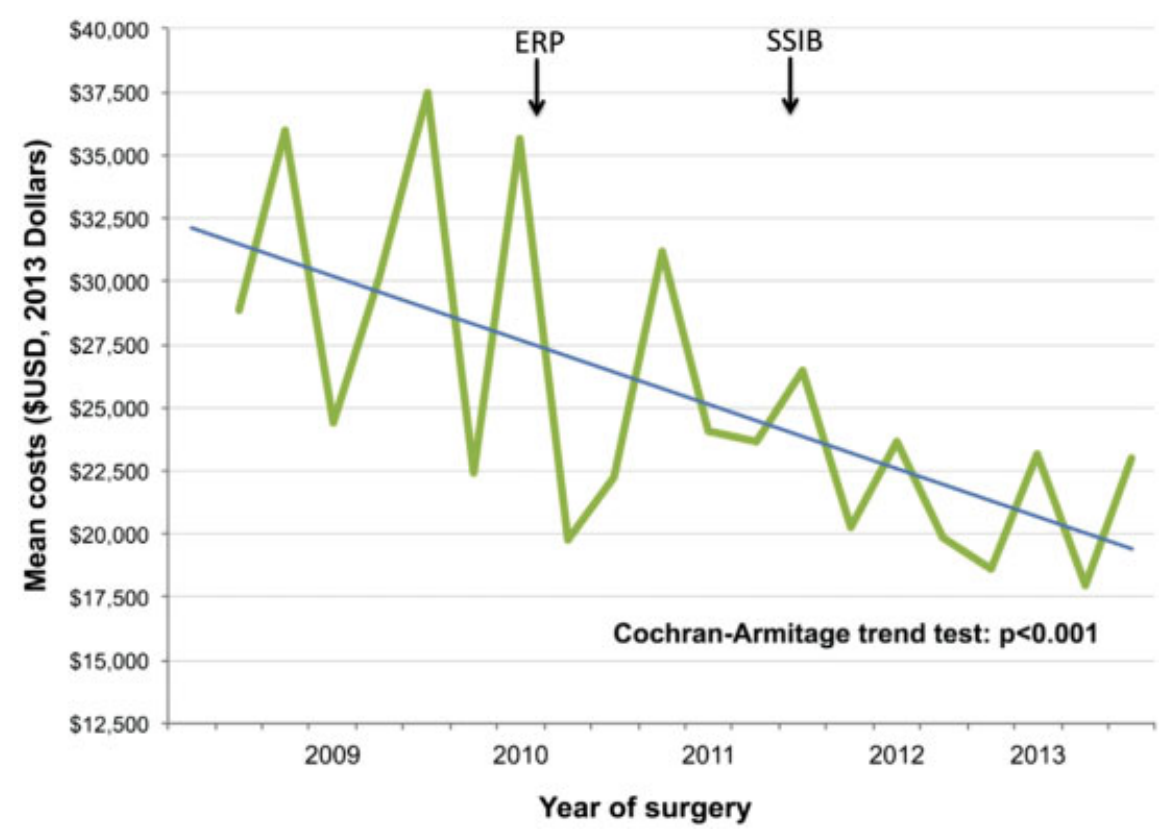

Fig. 8 Mean costs per admission US Dollar (USD 2013) following implementation of enhanced recovery pathway (ERP) in 2010 and surgical site infection prevention bundle 2011 by Keenan et al (2015).

\section{Conflict of Interest}

The authors have no disclosure and received no financial support for their contributions to this article.

\section{References}

1 de Lissovoy G, Fraeman K, Hutchins V, Murphy D, Song D, Vaughn BB. Surgical site infection: incidence and impact on hospital utilization and treatment costs. Am J Infect Control 2009;37 (05):387-397

2 Umscheid CA, Mitchell MD, Doshi JA, Agarwal R, Williams K, Brennan PJ. Estimating the proportion of healthcare-associated infections that are reasonably preventable and the related mortality and costs. Infect Control Hosp Epidemiol 2011;32(02): $101-114$

3 Jha AK, Chan DC, Ridgway AB, Franz C, Bates DW. Improving safety and eliminating redundant tests: cutting costs in U.S. hospitals. Health Aff (Millwood) 2009;28(05):1475-1484

4 Bull A, Wilson J, Worth LJ, et al. A bundle of care to reduce colorectal surgical infections: an Australian experience. J Hosp Infect 2011;78(04):297-301

5 Hedrick TL, Heckman JA, Smith RL, Sawyer RG, Friel CM, Foley EF. Efficacy of protocol implementation on incidence of wound infection in colorectal operations. J Am Coll Surg 2007;205(03): 432-438

6 Keenan JE, Speicher PJ, Thacker JK, Walter M, Kuchibhatla M, Mantyh CR. The preventive surgical site infection bundle in colorectal surgery: an effective approach to surgical site infection reduction and health care cost savings. JAMA Surg 2014;149(10): 1045-1052

7 Landrigan CP, Parry GJ, Bones CB, Hackbarth AD, Goldmann DA, Sharek PJ. Temporal trends in rates of patient harm resulting from medical care. N Engl J Med 2010;363(22):2124-2134

8 Donaldson MS, Corrigan JM, Kohn LT. To Err is Human: Building a Safer Health System. Washington, DC: National Academies Press; 2000

9 National Nosocomial Infections Surveillance System. National Nosocomial Infections Surveillance (NNIS) System Report, data summary from January 1992 through June 2004, issued October 2004. Am J Infect Control 2004;32(08):470-485

10 Tokars JI, Richards C, Andrus M, et al. The changing face of surveillance for health care-associated infections. Clin Infect Dis 2004;39(09):1347-1352

11 Pope G. About ACS NSQIP. American College of Surgeons; 2017. Available at: https://www.facs.org/quality-programs/acs-nsqip/ about. accessed on January 14, 2019

12 Steinberg SM, Popa MR, Michalek JA, Bethel MJ, Ellison EC. Comparison of risk adjustment methodologies in surgical quality improvement. Surgery 2008;144(04):662-667, discussion 662-667

13 Bordeianou L, Cauley CE, Antonelli D, et al. Truth in reporting: how data capture methods obfuscate actual surgical site infection rates within a health care network system. Dis Colon Rectum 2017;60(01):96-106

14 Bratzler DW, Houck PM; Surgical Infection Prevention Guidelines Writers Workgroup; American Academy of Orthopaedic Surgeons; American Association of Critical Care Nurses; American Association of Nurse Anesthetists; American College of Surgeons; American College of Osteopathic Surgeons; American Geriatrics Society; American Society of Anesthesiologists; American Society of Colon and Rectal Surgeons; American Society of Health-System Pharmacists; American Society of PeriAnesthesia Nurses; Ascension Health; Association of periOperative Registered Nurses; Association for Professionals in Infection Control and Epidemiology; Infectious Diseases Society of America; Medical Letter; Premier; Society for Healthcare Epidemiology of America; Society of Thoracic Surgeons; Surgical Infection Society. Antimicrobial prophylaxis for surgery: an advisory statement from the National Surgical Infection Prevention Project. Clin Infect Dis 2004;38(12):1706-1715

15 Bratzler DW, Hunt DR. The surgical infection prevention and surgical care improvement projects: national initiatives to improve outcomes for patients having surgery. Clin Infect Dis 2006;43(03):322-330

16 Yokoe DS, Anderson DJ, Berenholtz SM, et al; Society for Healthcare Epidemiology of America (SHEA). A compendium of strategies to prevent healthcare-associated infections in acute care hospitals: 2014 updates. Infect Control Hosp Epidemiol 2014;35 (08):967-977 
17 Care TJCCfTH. SSI Infographic. 2010. Available at: http://www. centerfortransforminghealthcare.org/assets/4/6/SSI_abdominal_ wall_infographic.pdf. Accessed on January 14, 2019

18 Healthcare JCCfT. Reducing Colorectal Surgical Site Infections. 2017. Available at: http://www.centerfortransforminghealthcare. org/assets/4/6/SSI_abdominal_wall_infographic.pdf. Accessed on January 14, 2019

19 Smith RL, Bohl JK, McElearney ST, et al. Wound infection after elective colorectal resection. Ann Surg 2004;239(05):599-605, discussion 605-607

20 Wick EC, Hirose K, Shore AD, et al. Surgical site infections and cost in obese patients undergoing colorectal surgery. Arch Surg 2011; 146(09):1068-1072

21 Speicher PJ, Nussbaum DP, Scarborough JE, et al. Wound classification reporting in HPB surgery: can a single word change public perception of institutional performance? HPB 2014;16(12): 1068-1073

22 Bilimoria KY, Liu Y, Paruch JL, et al. Development and evaluation of the universal ACS NSQIP surgical risk calculator: a decision aid and informed consent tool for patients and surgeons. J Am Coll Surg 2013;217(05):833-42.e1, 3

23 Itani KM, Jensen EH, Finn TS, Tomassini JE, Abramson MA. Effect of body mass index and ertapenem versus cefotetan prophylaxis on surgical site infection in elective colorectal surgery. Surg Infect (Larchmt) 2008;9(02):131-137

24 Dellinger EP, Hausmann SM, Bratzler DW, et al. Hospitals collaborate to decrease surgical site infections. Am J Surg 2005;190 (01):9-15

25 Scarborough JE, Mantyh CR, Sun Z, Migaly J. Combined mechanical and oral antibiotic bowel preparation reduces incisional surgical site infection and anastomotic leak rates after elective colorectal resection: an analysis of colectomy-targeted ACS NSQIP. Ann Surg 2015;262(02):331-337

26 Classen DC, Evans RS, Pestotnik SL, Horn SD, Menlove RL, Burke JP. The timing of prophylactic administration of antibiotics and the risk of surgical-wound infection. N Engl J Med 1992;326(05): 281-286

27 Polk HC Jr, Christmas AB. Prophylactic antibiotics in surgery and surgical wound infections. Am Surg 2000;66(02):105-111

28 van den Berghe G, Wouters P, Weekers F, et al. Intensive insulin therapy in critically ill patients. N Engl J Med 2001;345(19): 1359-1367

29 Kurz A, Sessler DI, Lenhardt R; Study of Wound Infection and Temperature Group. Perioperative normothermia to reduce the incidence of surgical-wound infection and shorten hospitalization. N Engl J Med 1996;334(19):1209-1215

30 Sessler DI, Akça O. Nonpharmacological prevention of surgical wound infections. Clin Infect Dis 2002;35(11):1397-1404

31 Allen DB, Maguire JJ, Mahdavian M, et al. Wound hypoxia and acidosis limit neutrophil bacterial killing mechanisms. Arch Surg 1997;132(09):991-996

32 Greif R, Akça O, Horn EP, Kurz A, Sessler DI; Outcomes Research Group. Supplemental perioperative oxygen to reduce the incidence of surgical-wound infection. N Engl J Med 2000;342(03): 161-167

33 Itani KM, Wilson SE, Awad SS, Jensen EH, Finn TS, Abramson MA. Ertapenem versus cefotetan prophylaxis in elective colorectal surgery. N Engl J Med 2006;355(25):2640-2651

34 Sparling KW, Ryckman FC, Schoettker PJ, et al. Financial impact of failing to prevent surgical site infections. Qual Manag Health Care 2007; 16(03):219-225

35 Bliss LA, Maguire LH, Chau Z, et al. Readmission after resections of the colon and rectum: predictors of a costly and common outcome. Dis Colon Rectum 2015;58(12):1164-1173

36 Arriaga AF, Lancaster RT, Berry WR, et al. The better colectomy project: association of evidence-based best-practice adherence rates to outcomes in colorectal surgery. Ann Surg 2009;250(04): 507-513
37 Stulberg JJ, Delaney CP, Neuhauser DV, Aron DC, Fu P, Koroukian $\mathrm{SM}$. Adherence to surgical care improvement project measures and the association with postoperative infections. JAMA 2010; 303(24):2479-2485

38 Berríos-Torres SI, Umscheid CA, Bratzler DW, et al; Healthcare Infection Control Practices Advisory Committee. Centers for Disease Control and Prevention guideline for the prevention of surgical site infection, 2017. JAMA Surg 2017;152(08):784-791

39 World Health Organization. Global Guidelines on the Prevention of Surgical Site Infection. Geneva: WHO; 2016:184

40 Keenan JE, Speicher PJ, Nussbaum DP, et al. Improving outcomes in colorectal surgery by sequential implementation of multiple standardized care programs. J Am Coll Surg 2015;221(02): 404-14.e1

41 Zhuang CL, Ye XZ, Zhang XD, Chen BC, Yu Z. Enhanced recovery after surgery programs versus traditional care for colorectal surgery: a meta-analysis of randomized controlled trials. Dis Colon Rectum 2013;56(05):667-678

42 Berian JR, Ban KA, Liu JB, Ko CY, Feldman LS, Thacker JK. Adherence to enhanced recovery protocols in NSQIP and association with colectomy outcomes. Ann Surg 2017. Doi: 10.1097/ sla.0000000000002566

43 Kehlet H, Wilmore DW. Evidence-based surgical care and the evolution of fast-track surgery. Ann Surg 2008;248(02):189-198

44 Senagore AJ, Stulberg JJ, Byrnes J, Delaney CP. A national comparison of laparoscopic vs. open colectomy using the National Surgical Quality Improvement Project data. Dis Colon Rectum 2009;52(02):183-186

45 Schwenk W, Haase O, Neudecker J, Müller JM. Short term benefits for laparoscopic colorectal resection. Cochrane Database Syst Rev 2005;(03):CD003145

46 Schweizer ML, Cullen JJ, Perencevich EN, Vaughan Sarrazin MS Costs associated with surgical site infections in Veterans Affairs Hospitals. JAMA Surg 2014;149(06):575-581

47 Lee KK, Berenholtz SM, Hobson DB, Demski RJ, Yang T, Wick EC. Building a business case for colorectal surgery quality improvement. Dis Colon Rectum 2013;56(11):1298-1303

48 De Angelis G, Murthy A, Beyersmann J, Harbarth S. Estimating the impact of healthcare-associated infections on length of stay and costs. Clin Microbiol Infect 2010;16(12):1729-1735

49 Graves N, Barnett AG, Halton K, et al. The importance of good data, analysis, and interpretation for showing the economics of reducing healthcare-associated infection. Infect Control Hosp Epidemiol 2011;32(09):927-928, author reply 928-930

50 Broex EC, van Asselt AD, Bruggeman CA, van Tiel FH. Surgical site infections: how high are the costs? J Hosp Infect 2009;72(03): 193-201

51 Zimlichman E, Henderson D, Tamir O, et al. Health care-associated infections: a meta-analysis of costs and financial impact on the US health care system. JAMA Intern Med 2013;173(22):2039-2046

52 Urban JA. Cost analysis of surgical site infections. Surg Infect (Larchmt) 2006;7(Suppl 1):S19-S22

53 Wilson SE, Turpin RS, Kumar RN, et al. Comparative costs of ertapenem and cefotetan as prophylaxis for elective colorectal surgery. Surg Infect (Larchmt) 2008;9(03):349-356

54 Lee L, Mata J, Ghitulescu GA, et al. Cost-effectiveness of enhanced recovery versus conventional perioperative management for colorectal surgery. Ann Surg 2015;262(06):1026-1033

55 Dimick JB, Chen SL, Taheri PA, Henderson WG, Khuri SF, Campbell DA Jr. Hospital costs associated with surgical complications: a report from the private-sector National Surgical Quality Improvement Program. J Am Coll Surg 2004;199(04):531-537

56 Centers for Medicare and Medicaid Services (CMS), HHS. Medicaid program; payment adjustment for provider-preventable conditions including health care-acquired conditions. Final rule. Fed Regist 2011;76(108):32816-32838

57 Services CfMM. Hospital-Acquired Conditions. 2015. Available at: https://www.cms.gov/Medicare/Medicare-Fee-for-Service- 
Payment/HospitalAcqCond/Hospital-Acquired_Conditions. html. Accessed on: January 14, 2019

58 Waters TM, Daniels MJ, Bazzoli GJ, et al. Effect of Medicare's nonpayment for hospital-acquired conditions: lessons for future policy. JAMA Intern Med 2015;175(03):347-354

59 McNair PD, Luft HS, Bindman AB. Medicare's policy not to pay for treating hospital-acquired conditions: the impact. Health Aff (Millwood) 2009;28(05):1485-1493

60 Birkmeyer NJ, Birkmeyer JD. Strategies for improving surgical quality-should payers reward excellence or effort? $\mathrm{N}$ Engl J Med 2006;354(08):864-870

61 Rosenthal MB, Dudley RA. Pay-for-performance: will the latest payment trend improve care? JAMA 2007;297(07):740-744

62 Grol R, Grimshaw J. From best evidence to best practice: effective implementation of change in patients' care. Lancet 2003;362 (9391):1225-1230
63 Wick EC, Galante DJ, Hobson DB, et al. Organizational culture changes result in improvement in patient-centered outcomes: implementation of an integrated recovery pathway for surgical patients. J Am Coll Surg 2015;221(03):669-677, quiz 785-786

64 Hechenbleikner EM, Hobson DB, Bennett JL, Wick EC. Implementation of surgical quality improvement: auditing tool for surgical site infection prevention practices. Dis Colon Rectum 2015;58 (01):83-90

$65 \mathrm{Vu} \mathrm{JV}$, Collins SD, Seese E, et al. Evidence that a regional surgical collaborative can transform care: surgical site infection prevention practices for colectomy in Michigan. J Am Coll Surg 2018;226 (01):91-99

66 Lee L, Li C, Landry T, et al. A systematic review of economic evaluations of enhanced recovery pathways for colorectal surgery. Ann Surg 2014;259(04):670-676 